\title{
QCD: Results from Lattice Quantum Chromodynamics
}

Quantum chromodynamics (QCD) is the modern theory of the strong force. In this theory, the main objects are quarks and gluons, which are bound by the strong force into protons, neutrons, and other particles called hadrons. In the framework of QCD, the strong nuclear force binding protons and neutrons together into nuclei is actually only a residue of the much stronger forces acting between quarks and gluons. In fact, inside the proton, even the concept of force is not very useful. Within all hadrons we have a swirl of gluons being exchanged back and forth as a manifestation of the strong force. To make matters worse, gluons can split into two, and then rejoin, or they can split into a quark-antiquark pair. Even the simplest hadron is a complex system hosting constantly interacting components.

Despite this complexity, QCD is well established experimentally. This is because at short distances (or high energies), the coupling between the particles is effectively small and particles move around with relative freedom. This is called asymptotic freedom and QCD is amenable to the traditional methods of quantum field theory in this regime. High-energy experiments have tested and confirmed QCD in this realm, which led to the 2004 Nobel Prize in Physics for Drs. David Gross, David Politzer, and Frank Wilczek, the theorists who provided the theory for short-range QCD and asymptotic freedom.

On the other hand, the traditional methods break down when gluons (and all the complexities that they entail) travel over "long" distances (to particle physicists, the nuclear size $10^{-15} \mathrm{~m}$ is a long distance). There is a mathematically rigorous way to handle the gluons in such cases, which is to introduce a grid in space-time - the "lattice" of lattice QCD - and use a computer to keep track of the quarks and gluons on this grid. Of course, the computer does not do everything; the person running the computer has to make sure that the results do not depend on how fine or coarse the grid is. Fortunately, the grid spacing is considered a "short" distance, so on this scale we can use the well-tested methods of perturbative (short distance) QCD.

As is the case for all scientific and computational problems, results need to be tested and validated and we need to be sure that the techniques employed are correct. Physicists from three lattice QCD collaborations (Fermilab Lattice, MILC, and, HPQCD collaborations) decided to address these concerns by making some specific predictions. We used the same methods and input parameters that were used previously with successful results, and set out to calculate the properties of some processes whose measurements were just around the corner

Our first prediction was for a process very similar to that shown in figure 1 . Instead of a B meson the initial state is a D meson, and the final hadron is now a kaon $(K)$. In this case the underlying quark decay is charm to strange ( $->s$ ), which is better understood than $b$ $\longrightarrow \mathrm{u}$. If we denote the total momentum of the final state electron $(\mathrm{e})$ and neutrino $(\mathrm{V})$ in $\mathrm{D} \rightarrow \mathrm{KeV}$ as $\mathrm{q}$, then we predicted that the $\mathrm{c}-$ >s decay rate should depend on $q^{2}$. The $q^{2}$ dependence had not been measured when our paper was submitted. Two months later, an experiment called FOCUS published the first measurement of the $q^{2}$ dependence of this decay and confirmed our calculation. Since then, the Belle collaboration has completed an even more precise experimental measurement. A comparison between measurement and prediction for the QCD part of the decay (also called the form factor) is shown in figure 2. Our calculation is shown as a curve with an error band, and it is clear that the data points from the Belle experiment fall right on top of this curve.

Another prediction that we made was for a simpler decay of the $D$ meson into just a positron and a neutrino with no daughter hadron. Here a single number, called the decay constant, summarizes the influence of QCD on this process. There are actually two mesons of interest, the $D^{+}$and the $D_{S}$; the latter has a strange (s) quark in it. We finished our work on this decay in June 2005 and the CLEO experiment confirmed our calculation at a conference that July. Since then, another experiment named BaBar has released the first precise measurement of the $D_{S}$ decay constant. This also agrees with our prediction. A nice way to summarize these results is to take the ratio of the two decay constants, because then several uncertainties from the lattice QCD approach cancel. We predicted that the ratio should be $0.786 \pm 0.042$, using lattice $Q C D$, and the experiments described above have found a ratio of $0.779 \pm 0.093$ 


\section{Long Range Gluons of QCD}

There are many problems in particle physics, nuclear physics, and astrophysics that require a quantitative understanding of the connection between quarks and gluons. One of the most basic problems in particle physics, for example, is the experimental quest to understand the interactions between each kind of quark. There are known to be six different types: down, up, strange, charm, bottom, and top. Most of the world is made of up and down quarks, which are confined inside protons and neutrons. But to particle physicists the others are more interesting, because their interactions are intimately related to the origin and evolution of the universe.

The situation described so far is depicted in figure 1 . We would like to understand the decay of a bottom (or b, for short) quark, for example, but the b quarks are usually found inside another particle called the B meson. In this environment the $b$ quark will decay to an up (u) quark, and emit (through a virtual $\mathrm{W}$ boson) an electron and an antineutrino. No existing experimental apparatus can detect a free quark; instead, we detect the hadron (such as a pion, O) which contains the quark resulting from the decay.

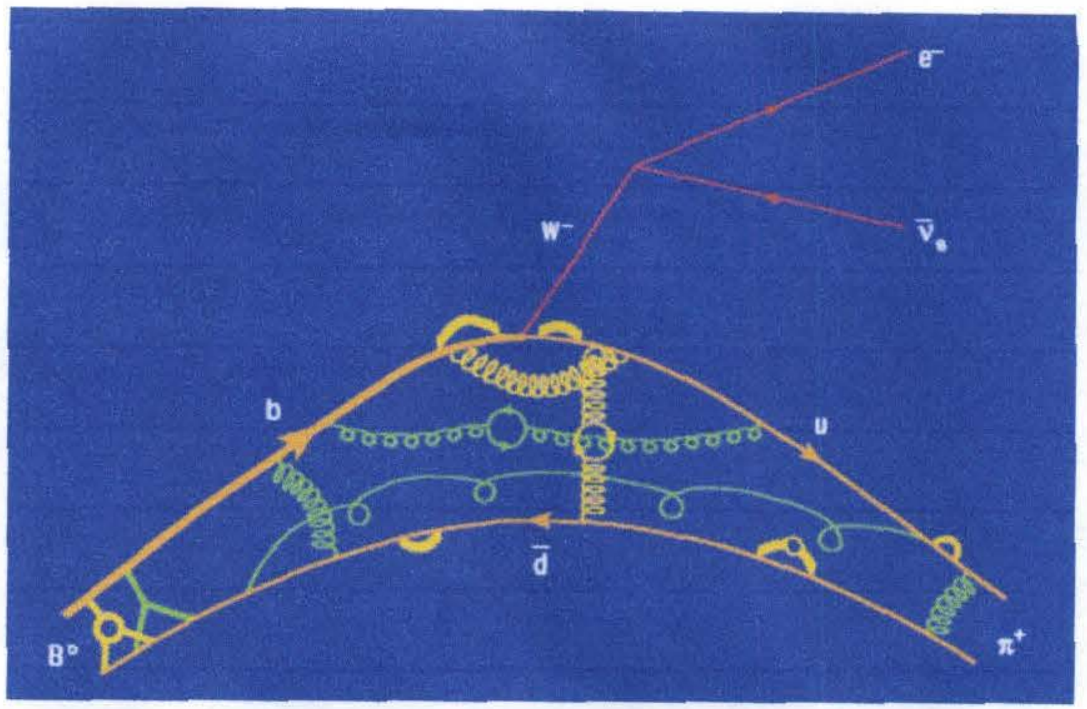

Figure 1. The decay of a neutral B meson. The weak interaction sector (particles connected to the $W$ boson) and the short-range gluons (short coiled lines) can be handled with traditional methods of quantum field theory. The long-range gluons (long coiled lines) need a numerical technique, known as lattice QCD. Recent advances in lattice QCD now include a realistic treatment of the subprocesses in which a gluon turns into a quark loop and back into gluons again.

The principal challenge of QCD is to work out the strong, nonlinear interactions of long-range gluons. The best approach, which is already over 25 years old, is to use the lattice formulation of QCD and calculate the interactions on a computer. For many years, computing resources were not good enough to simulate one important aspect of these interactions: the tendency of gluons to turn into quark-antiquark pairs, then back into gluons. The situation finally changed at the beginning of this decade, when a combination of insightful ideas and gains in computing power permitted precise calculations of hadron properties. The predictions that resulted from this work agree with experimental measurements to an accuracy of about $1 \%$ or $2 \%$.

The last prediction that we would like to discuss is the mass of an exotic particle called the charmed beauty meson, or $\mathrm{B}_{\mathrm{C}}$. The calculation of its mass tests two aspects of QCD: on one level, our methods for treating the interaction between charmed and bottom quarks; and on another level, our methods for treating the conversion of gluons into light quark pairs. Our calculation obtained a mass of $6,304 \pm 20 \mathrm{MeV} / \mathrm{c}^{2}$, and was completed before any experiment succeeded in the measurement. In this case, the CDF experiment found a mass of $6,287 \pm 5 \mathrm{MeV} / \mathrm{c}^{2}$. Figure 3 shows an interesting comparison with previous results. The point on the left is an older calculation that did not include the conversion of gluons into quark pairs and back again. While its range includes the experimental result, it is not constrained enough. It demonstrates that the heavy quarks (charmed and bottom) were being included correctly, but that some other sections may be missing. The modern calculation (middle point) corrects this discrepancy, and is in good agreement with the measurement from the CDF experiment (right-hand point).

Taken together, these results represent a stringent test of lattice QCD. As in any numerical simulation, there are several ingredients going into our computer code, and each of the three predictions probes a different aspect of the contributing physics. Furthermore, the $\mathrm{D}$ decay properties - the leptonic decay constant and the semileptonic form factors-can now be easily adapted to calculate the analogous properties of $\mathrm{B}$ mesons, simply by changing the mass of the heavy quark when we run the simulation. 


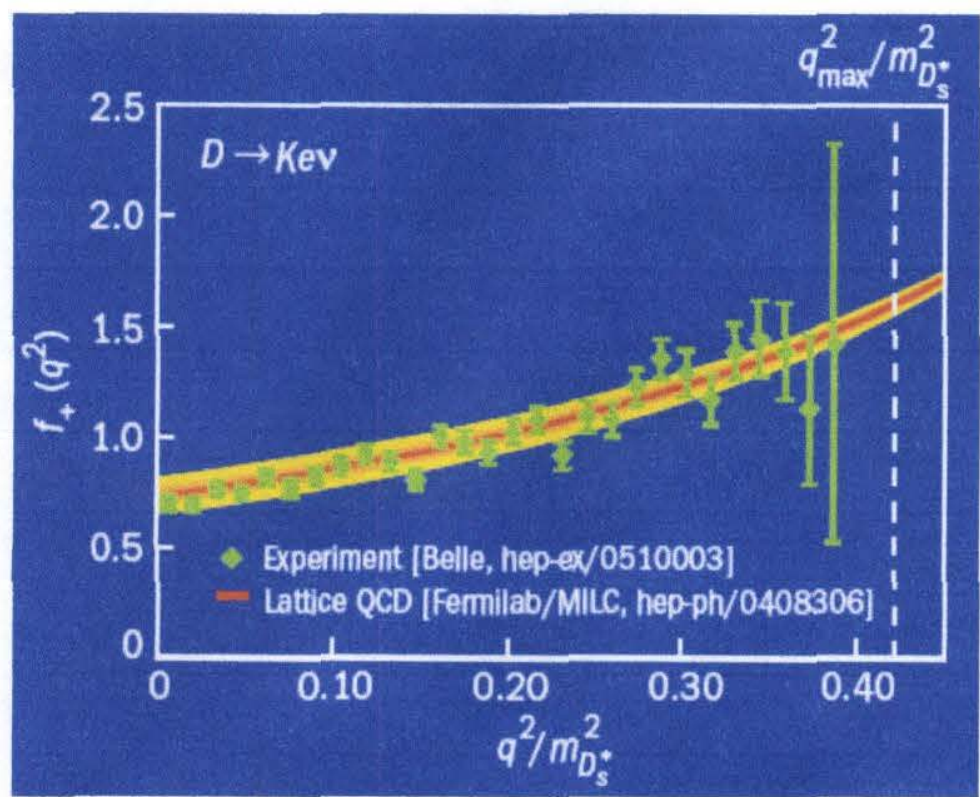

Figure 2. Comparison of the $q 2$ dependence of the form factor in the decay $D \rightarrow K e n$. The error band shows a calculation using lattice $Q C D$ and the points give an experimental measurement from the Belle Collaboration.

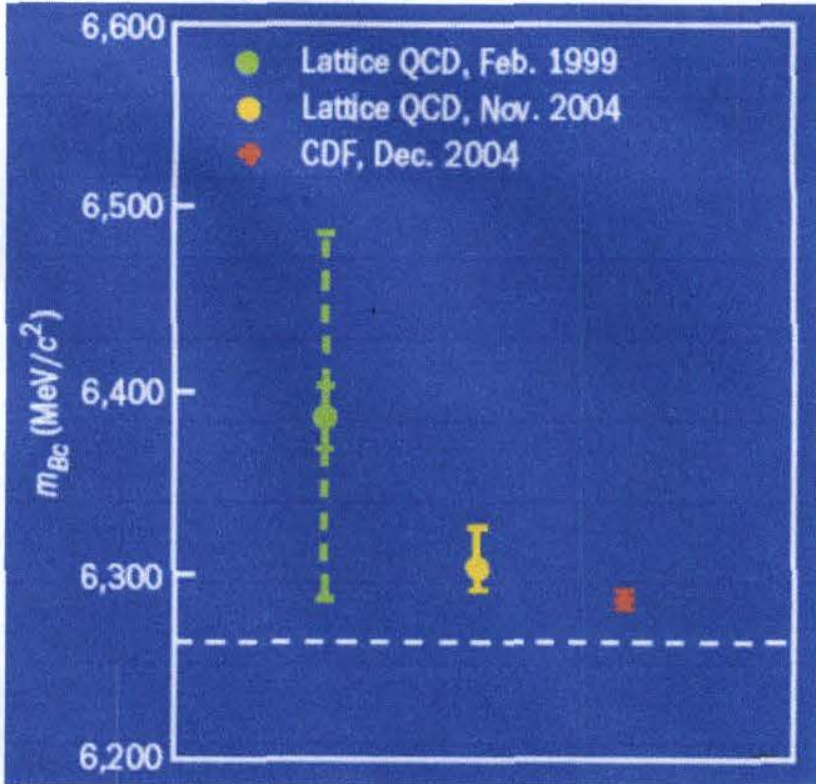

Figure 3. Comparison of the Bc meson mass. From left to right: an old lattice calculation omitting the conversion of gluons into quarks and back; a modem calculation this feature of QCD; an experimental measurement from the $C D F$ Collaboration.

The B meson is one of hottest topics in all of elementary particle physics, and is being subjected to intense scrutiny by experiments at several particle accelerator laboratories. The reason for this intensive study is that the underlying interactions of the $b$ quark are thought to provide important clues about the nature of the universe. How do quarks attain their mass? Or, an even more intriguing question: which elementary processes are responsible for the abundance of matter (and dearth of antimatter) in the universe? The properties of the different quark flavors are connected to yet another mind-boggling question: why is the up quark's mass a little bit smaller than the down quark's mass? If it were the other way around (which would fit the pattern seen in more exotic quarks), then protons would decay to neutrons instead of vice versa. A universe with this property would be made only of neutrons, neutrinos, and photons; there would be no atoms, no chemistry, and no life.

The answers to some of these questions may be close at hand. If any of the relevant effects of QCD on $B$ meson decays are large enough, then terascale lattice QCD with an accuracy of $1 \%$ or $2 \%$ could begin to disentangle the underlying interactions. The harder questions in quark flavor physics-which are related to other overarching questions in particle physics, nuclear physics, and astrophysics-will require even more ambitious numerical calculations at the petascale. Our collaboration is well positioned to proceed to the challenges and promises of petascale computing. Quantum chromodynamics is one of the most powerful theories of the physical world and the combination of innovative minds and advanced computing resources can utilize its full potential to unravel more of the secrets of science at ultrasmall and ultra-large scales.

Contributor: Dr. Andreas S. Kronfeld, Theoretical Physics Department, Fermi National Accelerator Laboratory

PI: Dr. Robert Suger, University of California-Santa Barbara; work done under the Lattice QCD SciDAC Project 